\title{
Comparativo da técnica direta e semidireta no manejo das lesões cervicais dentárias não cariosas: relato de caso
}

\author{
Comparison of direct and semi-direct technique in the management of non-carious cervical \\ dental lesions: case report
}

Comparación de la técnica directa y semidirecta en el tratamiento de las lesiones cervicales dentales no cariosas: reporte de caso

Maria Amanda Pereira Rêgo ${ }^{1}$, Karlos Eduardo Rodrigues Lima1, Eduardo da Cunha Queiroz ${ }^{1}$, Érika Matias Pinto Dinelly ${ }^{1}$, Thayla Hellen Nunes Gouveia ${ }^{1}$, Jossaria Pereira de Sousa ${ }^{2}$, Natasha Muniz Fontes $^{1}$, Julianne Coelho da Silva ${ }^{3}$, Talita Arrais Daniel Mendes ${ }^{1,3 *}$, Luiz Filipe Barbosa Martins ${ }^{1,4}$.

\section{RESUMO}

Objetivo: Avaliar clinicamente as diferenças entre as técnicas restauradoras direta e semidireta com uso de resina composta, em dentes com lesões cervicais não cariosas (LCNC) por abfração, nos critérios de: retenção, integridade marginal, descoloração marginal e satisfação do paciente. Detalhamento de caso: Trata-se de um relato de caso, no qual foi selecionada uma paciente de demanda livre que apresentava pelo menos 4 LCNC, 2 em cada hemi-arco, assim sendo utilizou o modelo "splitmouth". As LCNC foram restauradas por 2 protocolos distintos, técnica direta e semidireta com o uso de resina composta nanoparticulada, onde 2 dentes foram restaurados com uma técnica e outros 2 com outra, após a realização dos protocolos foram avaliados parâmetros clínicos descritos, imediatamente e após 30 dias afim de estabelecer um protocolo de melhor longevidade e estética. Considerações finais: As restaurações realizadas através da técnica direta mostraram-se mais eficazes para as restaurações em LCNCs, quando compradas à técnica semidireta, durante o período avaliado.

Palavras-chave: Dentina, Reabilitação bucal, Restaurações intracoronárias.

\section{ABSTRACT}

Objective: Evaluate clinically how differences between direct and semi-direct restorative techniques with the use of composite resin, in teeth with non-carious cervical lesions (LCNC) due to friction, in the requirements of: retention, marginal integrity, marginal discoloration and patient satisfaction. Case detail: It is a case report, in which a patient was selected where a patient with free demand was selected who had at least 4 LCNC, 2 in each hemi-arch, thus using the split mouth model. The LCNC were restored by 2 different protocols, direct and semi-direct technique using nanoparticulate composite resin, where 2 teeth were restored with one technique and another 2 with another, after performing the protocols, parameters previously described were evaluated, immediately and after 30 days in order to establish a protocol of better longevity and aesthetics. Final considerations: Restorations performed using the direct technique proved to be more effective for restorations in LCNCs, when purchased using the semi-direct technique, during the evaluated period.

Key words: Dentin, Oral rehabilitation, Inlay.

\footnotetext{
${ }^{1}$ Centro Universitário Católica de Quixadá (Unicatólica), Quixadá - CE. *E-mail: talita_arrais@hotmail.com

2 Universidade Estadual da Paraíba (UEP), João Pessoa - PA.

${ }^{3}$ Universidade Federal do Ceará (UFC), Fortaleza - CE.

${ }^{4}$ Faculdade Paulo Picanço (FACPP), Fortaleza - CE.
} 


\section{RESUMEN}

Objetivo: Evaluar clínicamente las diferencias entre las técnicas de restauración directa y semidirecta con el uso de resina compuesta, en dientes con lesiones cervicales no cariosas (LCNC) debido a la fricción, en los criterios de retención, integridad marginal, decoloración marginal y satisfacción del paciente. Detalle del caso: Es un informe de caso, en el que se seleccionó un paciente en el que se seleccionó un paciente con demanda libre que tenía al menos 4 LCNC, 2 en cada hemi-arco, utilizando así el modelo de "boca dividida". Los LCNC se restauraron usando 2 protocolos diferentes, técnica directa y semidirecta usando resina compuesta nanoparticulada, donde se restauraron 2 dientes con una técnica y otros 2 con otra, después de realizar los protocolos, los parámetros clinicos se evaluaron inmediatamente y después de 30 días para establecer un protocolo de mejor longevidad y estética. Consideraciones finales: Las restauraciones realizadas utilizando la técnica directa demostraron ser más efectivas para restauraciones en LCNC, cuando se compraron utilizando la técnica semidirecta, durante el período evaluado.

Palabras clave: Dentina, Rehabilitación oral, Restauraciones intracoronarias.

\section{INTRODUÇÃO}

$\mathrm{Na}$ Odontologia restauradora, tem-se percebido um grande enfoque nos estudos de Lesões Cervicais Não Cariosas (LCNC), pelo fato dessas estarem cada dia mais presentes nos consultórios odontológicos e associadas aos hábitos alimentares e parafuncionais, o que tem influenciado diretamente no aumento da incidência deste tipo de lesões (MARSON FC, et al., 2017).

As LCNC são de origem multifatorial e podem ser classificadas como erosão, quando ocorre um desgaste químico dental proveniente de alguns ácidos não cariogênicos; abrasão, que pode ser de origem mecânica; e por fim, abfração que consiste na fratura da região cervical, sendo originada por forças mal distribuídas ao longo eixo do dente (MARSON FC, et al., 2017).

A LCNC pode ocorrer através de um processo fisiológico normal do corpo humano, mas ocorre também através de um fator patológico, gerando diversos graus de destruição dental e ocasionando sérios problemas funcionais, estéticos, e em alguns casos, a hipersensibilidade dentária (AMARAL SM, et al., 2012).

A principal característica das LCNC é uma perda de estrutura na região da junção amelocementária, sem envolver qualquer tipo de processo carioso (CARLO B, et al., 2017). Se não tratadas, podem acarretar em sintomas de dor de curta duração, como hipersensibilidade dentária. Isso acontece porque ocorre uma exposição de dentina, em decorrência da perda de esmalte ou devido às recessões gengivais que podem estar presentes também em alguns casos (GONÇALVES PE e DEUSDARA ST, 2012).

A devida escolha do material e técnica restauradora é de suma importância para o sucesso da restauração, porém, é interessante ressaltar que o controle e a resolução dos fatores etiológicos são o ponto primordial para a longevidade do tratamento (SOUSA LX, et al., 2018).

Esse relato de caso visou avaliar clinicamente as diferenças entre as técnicas restauradoras diretas e semidireta com o uso de resina composta em ambas as técnicas, em dentes com lesões cervicais não cariosas por abfração, no que se refere à: integridade marginal, descoloração marginal, retenção da restauração e satisfação do paciente.

\section{DETALHAMENTO DO CASO}

O presente estudo foi aprovado pelo comitê de ética em pesquisa sob o parecer de número 3.541.906. Paciente M.M.M.N. 52 anos, cor negra, sexo feminino, procurou atendimento odontológico. Foi realizado a anamnese, coletando-se dados relacionados à paciente, como: nome, data de nascimento, naturalidade/nacionalidade, história médica e odontológica, além, de sua queixa principal: "sinto muita dor em alguns dentes quando tomo qualquer coisa gelada, e esses desgastes no pé do dente me causam dor ao usar uma prótese". 
Ao exame clínico, foram observadas perda de estrutura dentária na região cervical dos dentes: 31(1 $\mathrm{mm}$ de profundidade), 34 ( $2 \mathrm{~mm}$ de profundidade) $43(1 \mathrm{~mm}$ de profundidade), 44 ( $2 \mathrm{~mm}$ de profundidade), o que são características clínicas das LCNC e presença de recessão gengival associada à lesão do dente 44 e 34 (Figura 1). Posteriormente, foi preenchido uma ficha clínica de coleta de dados onde se obteve alguns dados como informações sobre hábitos de higiene oral sensibilidade, tipo de dieta, presença de hábitos parafuncionais e tempo que percebeu a presença de LCNC. Todas as respostas aos questionamentos foram positivas.

Figura 1 - Aspecto inicial do paciente ao realizar a anamnese.

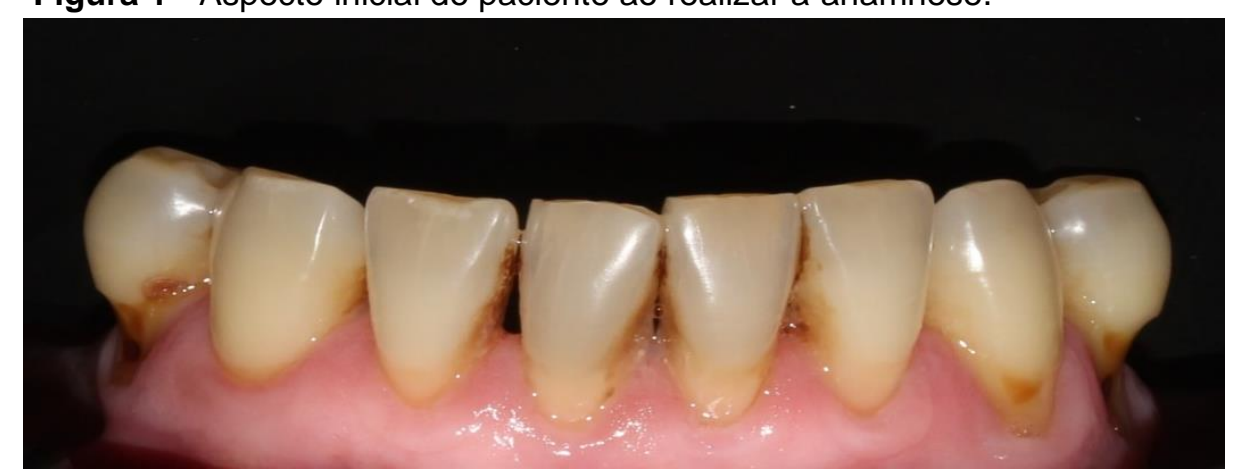

Fonte: RÊGO MAP, et al., 2020.

O caso clínico foi realizado seguindo o modelo boca dividida, para cada que hemi-arco recebesse um tratamento diferente, onde os elementos 31 e 34 receberam como técnica restauradora a técnica direta e os elementos 43 e 44 a técnica semidireta.

$\mathrm{Na}$ técnica direta realizada seguiu-se o protocolo que previamente à restauração foi realizada uma profilaxia com escova de Robinson (American Burrs), pedra- pomes e água, efetuando a limpeza da superfície dentária, realizou-se a seleção de cor, onde utilizou-se a resina composta Z350A3,5 "body" (3M ESPE) pela técnica incremental.

Realizou-se Isolamento relativo com afastador labial tipo Expandex (Maquira), rolete de algodão, sugador descartável (SSPlus), e fio retrator gengival Pro Retract 000 (FGM) afim de manter o ambiente livre de saliva evitando assim qualquer interferência nos procedimentos adesivos. Posteriormente realizou-se obisel com ponta diamantada de número 2200 (KG Sorensen) para mascarar alinha de união entre o dente e o material restaurador (Figura 2A). Dando sequência realizou-se condicionado seletivo do esmalte ácido fosfórico 37\% (Maquira- Maringá, Paraná, Brasil), por 30 segundos, a seguir lavou-se e secou-se (Figura 2B);

Figura 2 - Bizel realizado com ponta diamantada montada em alta rotação (A) e condicionamento seletivo de esmalte com ácido fosfórico a $37 \%$ por 30 segundos (B).

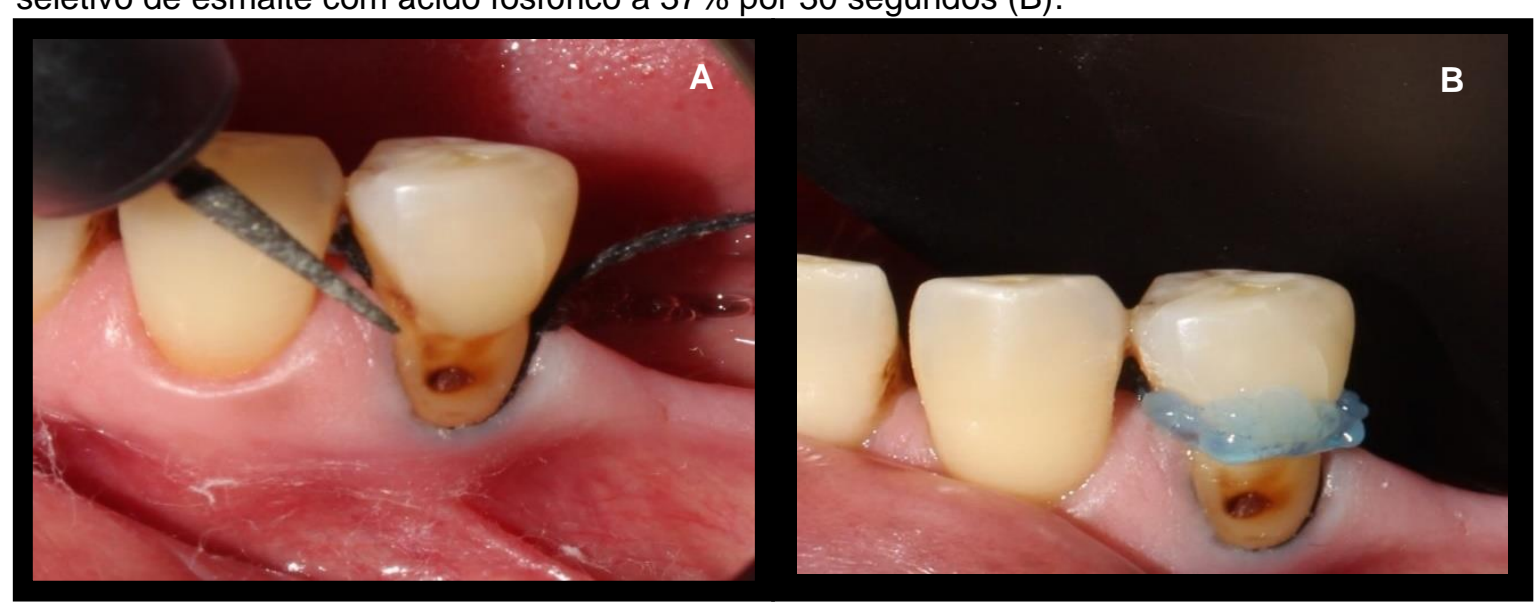

Fonte: RÊGO MAP, et al., 2020. 
Efetuou-se a aplicação do sistema adesivo autocondicionate (Clearfil Se Bond -3M ESPE) de forma ativa, com o auxílio de um micro-aplicador descartável Cavibrush (FGM) por 20 segundos. A seguir foi aplicado um jato de ar para evaporação do solvente, seguido de fotopolimerização (Ultralumen) por 40 segundos, realizouse incrementos de resina composta Z350A3,5 (3M ESPE), seguida de fotopolimerização por 20 segundos em cada incremento.

O acabamento foi realizado com ponta diamantada com granulação específica para acabamento montada em alta rotação, além de discos de lixa do tipo Sof-Lex Pop On (3M ESPE, Sumaré, São Paulo, Brasil). O polimento foi feito com pasta diamantada Diamond Gloss, (KG Sorensen Cotia, São Paulo, Brasil) e discos de feltro (FGM, Joinvile, Santa Catarina), montados em baixa rotação (Figura 3).

Figura 3 - Aspecto final das restaurações utilizando técnica semidireta.

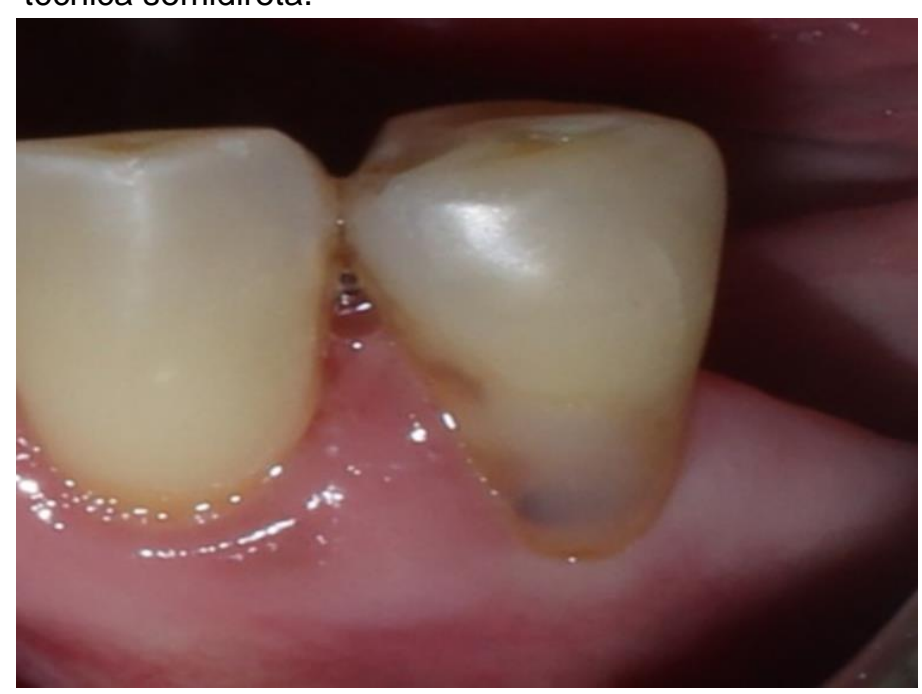

Fonte: RÊGO MAP, et al., 2020.

$\mathrm{Na}$ técnica semidireta realizada nos elementos 43 e 44 seguiu-se o seguinte protocolo de isolamento relativo e biselamento, seguido de moldagem da hemi-arcada dos elementos dentários com o auxílio de uma moldeira parcial , utilizando o silicone de adição (3M ESPE) primeiramente foi aplicado a pasta leve em região de margem cervical dos dentes que iriam receber as restaurações, a fim de obter uma cópia fidedigna de toda a margem cervical,a seguir foi colocado a pasta densa na moldeira parcial,então foi realizado a moldagem (Figura 4A) e ao final aguardou-se 1 hora para que fosse realizado o vazamento do molde na qual foi utilizado gesso especial tipo IV (Herostone -Coltene).

No modelo de gesso (Figura 4B) foi aplicado vaselina. A peça foi confeccionada utilizando a técnica incremental devolvendo a anatomia cervical de cada dente correspondente com uso de espátula Suprafill e pincel pelo de marta (Kota), deixando todas as superfícies lisas e livres de irregularidades. Ao final cada peça recebeu fotopolimerização de 40 segundos. As peças receberam polimerização adicional sendo colocadas dentro de um recipiente com água no forno micro-ondas por 5 minutos, após isso foi realizado o acabamento das margens da restauração em ambas as peças (Figura $\mathbf{4 C}$ ).

As superfícies internas das peças foram condicionadas (Figura 4D) com gel de ácido fosfórico 37\% (Maquira), por 30 segundos seguido de lavagem abundante com seringa tríplice e secagem com papel absorvente, posteriormente, com o auxílio de um micro aplicador descartável Cavibrush (FGM), aplicou-se o silano (Prosil- FGM) como agente de união da restauração por 20 segundos seguido de fotoativação (Ultralumen SL).

Para cimentação da peça, colocou-se fio retrator (Figura 4E) utilizou-se o cimento autoadesivo Rely XU200(3M ESPE) no interior da peça, a seguir posicionou-se nas cavidades e fotoativou-se (Ultralumen SL) por 40 segundos. $O$ acabamento foi realizado com ponta diamantada de granulação específica para acabamento montada em alta rotação, além de discos de lixa do tipo Sof-Lex Pop On (3M ESPE). O polimento foi feito com pasta diamantada Diamond Gloss (KG Sorensen) e discos de feltro (FGM) (Figura 4F). 
Figura 4 - Moldagem dos dentes com silicone de adição leve e pesado (A). Após o vazamento do molde (B), foi realizada a restauração com resina composta sobre o modelo de gesso (C). Preparação da peça com ácido fosfórico (D) seguido de silano e cimentação nos dentes (F).

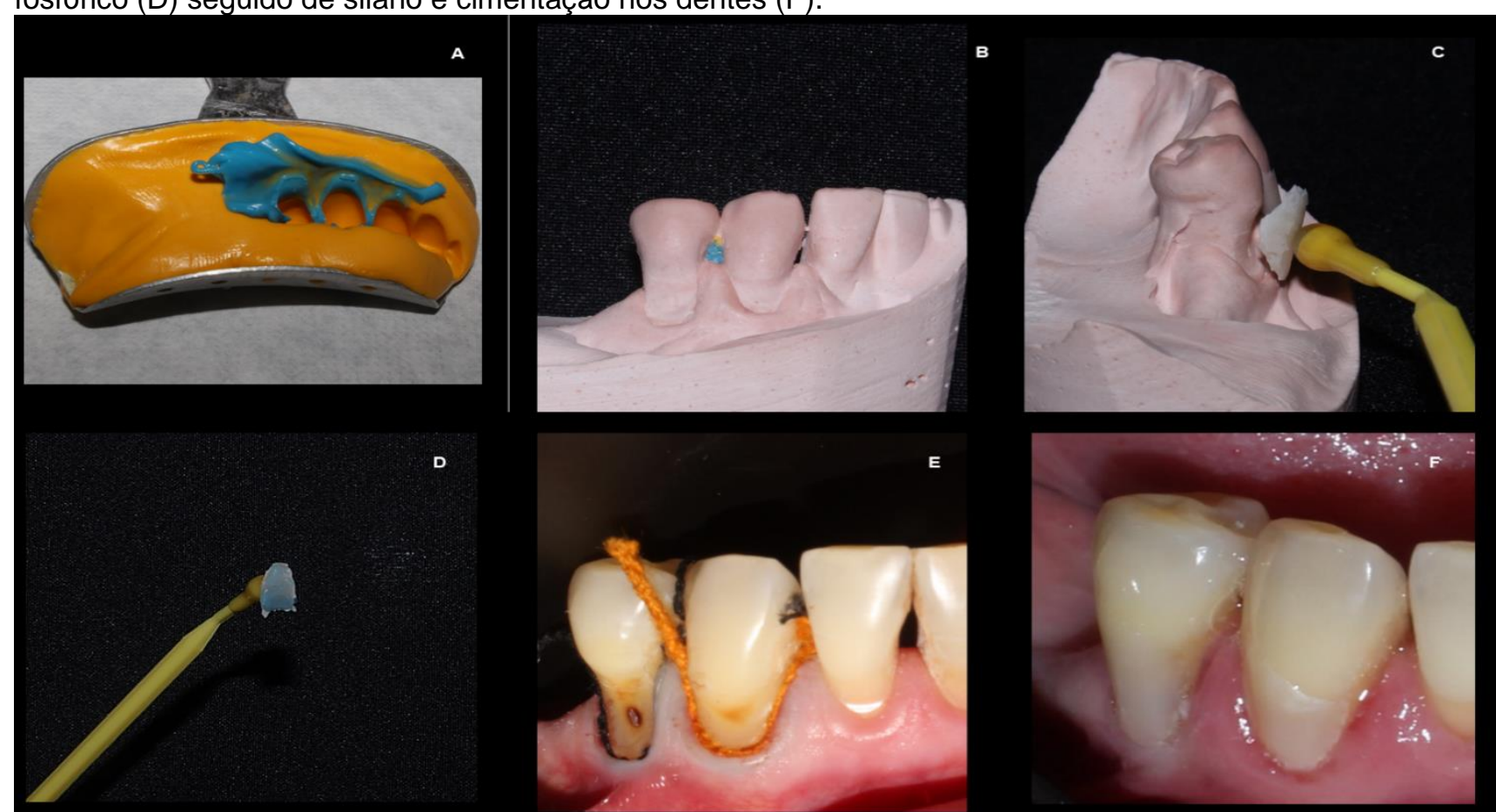

Fonte: RÊGO MAP et al., 2020.

\section{DISCUSSÃO}

Quando se leva em consideração a saúde dentária a longo prazo, as LCNC têm se tornado cada vez mais comuns, e a cada dia essa prevalência só aumenta (PEREZ CR, et al.,2012). A região cervical se torna mais propícia ao aparecimento de LCNC pelo fato de conter um baixo diâmetro coronário, o que somado às propriedades vítreas do esmalte, torna-se uma área de maior fragilidade aos agentes abrasivos e erosivos, e às forças de carga excessivas, favorecendo a presença de LCNC (GONÇALVES PE e DEUSDARA ST, 2012).

O estresse e os hábitos parafuncionais tem influenciado diretamente no aumento da incidência dessas lesões (MARSON FC, et al., 2017; SPLIETH CH e TACHOU A, 2013; BRANDINI DA, et al., 2011). Contudo, descobrir quais são os fatores etiológicos das LCNC é essencial para poder tratá-las, e por fim, poder então realizar o devido tratamento restaurador (GONÇALVES PE e DEUSDARA ST, 2012).

Em relação aos hábitos alimentares, observou-se uma dieta rica em alimentos ácidos e vegetarianas, mostraram maior vulnerabilidade ao surgimento de LCNC sem pessoas que tem esse hábito (KUMAR S, et al., 2015). Oliveira LKM et al. (2013) acrescentou ainda que as LCNCs estão relacionadas ao estilo de vida da população, no que se diz respeito à dieta ácida e ao grande consumo de industrializados. Hábitos de escovação vertical também estão correlacionadas ao surgimento de LCNC (GONÇALVES PE e DEUSDARA ST, 2012).

Percebe-se, através do relatado pela paciente, que ela realizava escovação de modo inapropriado e com a utilização de uma escova de cerdas duras, pois achava ser o correto, corroborando com as afirmações dos autores acima mencionados.

Quando as lesões já se encontram em um grau mais avançado se faz necessário o tratamento restaurador, juntamente com orientação de hábitos de higiene e dieta ao paciente, a fim de prevenir futuras lesões(PECIE $\mathrm{R}$, et al., 2011).Na maioria dos casos, pode ser necessário o procedimento restaurador, a fim de diminuir o problema de hipersensibilidade e comprometimento estético causado por essas lesões (TUNCER D, et al., 2014). No presente caso a paciente apresentava lesões de tamanho significativo associado à sensibilidade dolorosa em decorrência das lesões maiores que já estavam causando exposição dentinária, afetando a estética. 
Para a realização da escolha do material que será utilizado, a preocupação com a durabilidade a longo prazo é algo significativo (KIM KL, et al., 2013). Em virtude de suas excelentes propriedades estéticas e boa longevidade, as resinas compostas tem sido o material de escolha e o mais indicado na maioria dos casos, por apresentar um ótimo desempenho clínico se comparada a outros materiais (PECIE R, et al., 2011; KIM $\mathrm{KL}$, et al.,2013). Entretanto, a capacidade de união das resinas só será efetiva e durável se a escolha do sistema adesivo for feita adequadamente o que poderá influenciar no sucesso da restauração. Os sistemas adesivos mais indicados para LCNC tem sido os autocondicionantes (MODENA RA, et al., 2016).

Monteiro RV et al. (2017) ressaltou queas resinas têm se mostrado satisfatórias para restabelecer estética e função em restaurações diretas, apresentando boa sobrevida no geral, apresentando apenas taxa de falha anual de $1,8 \%$ após 5 anos, e 2,4\% após 10 anos.

Com relação à técnica restauradora para LCNCs, a direta tem sido a mais utilizada rotineiramente, por ser de simples e rápida execução, entretanto, alguns fatores como: ineficiente grau de conversão de monômeros em polímeros, luz e o fluxo salivar no momento da restauração tem sido apontado como algumas desvantagens inerentes à técnica (VAN DIJKEN JWV, 2000; NASCIMENTO GG, et al., 2013; ZORBA YO, et al.,2013).

Em contrapartida, Tuncer D et al. (2014) observou que longevidade tem sido um desafio, pois podem ocorrer falhas e comprometendo da restauração. Corroborando com Borges ALSet al. (2014), que afirmou que a contração de polimerização tem influenciado nos insucessos clínicos em restaurações diretas. Tonolli G e Hirata R (2010), Cavalheiro JP et al. (2014) e Godoy CE et al. (2014) afirmaram que um dos maiores motivos que tem levado ao fracasso as restaurações com resina composta é a contração de polimerização, que é diminuída através da técnica semidireta, devido a polimerização ocorrer extra oralmente, o que proporciona uma excelente estabilidade dimensional para a resina.

A técnica semidireta possui vantagens adicionais, como excelência na anatomia da superfície a ser restaurada, melhor adaptação marginal, bem como altas temperaturas na qual a peça é submetida promove uma maior conversão de polimerização, o que irá melhorar significativamente as propriedades mecânicas da restauração(TONOLLI G e HIRATA R, 2010;MONTEIRO RV, et al., 2017), o que justifica o aquecimento da peça do presente caso em micro-ondas. Além disso, não há a influência de saliva e proporciona ao paciente um maior conforto (TURBINO ML, et al.,2011; GARCÊS JF, 2011). No entanto, o presente estudo não observou os fatores mencionados, possuindo pior desempenho clínico.

No entanto, estudos mostram que não há diferença entre ambas as técnicas ao longo do tempo (VAN DIJKEN JWV, 2000; SPREAFICO RC, et al.,2005; SOUZA FHCD, 2006), indo de encontro com o presente caso, que demonstrou que a técnica direta foi mais efetiva. $O$ presente relato de caso entra em concordância com o trabalho realizado por Vieira RM et al. (2013) e Fahl-Jr N (2015), que observaram que a técnica direta tem se mostrado eficaz a longo prazo no tratamento restaurador de LCNC, sendo uma técnica rápida e de simples execução, sendo a técnica de primeira escolha e a mais utilizada no dia a dia nos consultórios odontológicos.

A restauração obtida pela técnica semidireta apresentou um menor mascaramento e menos integridade após 30 dias. Acredita-se que a perda de retenção das restaurações semidiretas pode ter ocorrido devido à presença de dentina esclerótica, o que pode ter dificultado a adesão do cimento. Luque-Martinez I et al. (2015), observou que o uso do ácido etilenodiamino tetra-acético aumenta a retenção de LCNC com adesivos autocondicionantes, o que pode justificar o que foi achado no presente relato, a presença de fendas após 30 dias poderia ser evitada com esse agente condicionante prévio.

O uso de um modelo boca-dividida permitiu a realização de duas técnicas restauradoras diferentes em uma única paciente, na qual esta foi submetida às mesmas condições críticas de higiene, escovação e alimentação inerentes à paciente.

Em relação ao tempo de acompanhamento e tamanho da amostra, foi possível concluir que, as restaurações realizadas através da técnica direta, se mostraram mais eficazes para o tratamento restaurador das LCNC, quando comparado à técnica semidireta no que se diz respeito à retenção das restaurações e consequentemente nos quesitos de descoloração e integridade marginal, durante o período avaliado, demandando um menor tempo clínico para o paciente e operador. 


\section{REFERÊNCIAS}

1. AMARAL SM, et al. Lesões não cariosas: o desafio do diagnóstico multidisciplinar. Arquivos Internacionais de Otorrinolaringologia, 2012; 16(1): 96-102.

2. BORGES ALS, et al. Impact of Quantity of Resin, C-factor, and Geometry on Resin Composite Polymerization Shrinkage Stress in Class V Restorations: Class V Cavity Design and Polymerization Stress. Operative Dentistry, 2014; 39(2): 144-151

3. BRANDINI DA, et al. Noncarious cervical lesions and their association with toothbrushing practices: in vivo evaluation. Operativa Dentistry, 2011; 36(6): 581-589.

4. CARLO B, et al. Microbiological characterization and effect of resin composites in cervical lesions. Journal of Clinical and Experimental Dentistry, 2017; 9(1): 40-45.

5. CAVALHEIRO JP, et al. Marginal adaptation of class $V$ restorations with current-generation dentin-bonding agents:effect of different dentin surface treatments. The journal of contemporary dental practice, 2014; 15(3): $331-335$

6. FAHL-JR N. Direct-Indirect C lass V Restorations: A Novel Approach for Treating Noncarious Cervical Lesions. JournalofEstheticandRestorativeDentistry, 2015; 27(5): 267-284.

7. GARCÊS JF. Técnica semi-direta para restauração de dente posterior - uma opção de tratamento. ApresentaçãoCentíficaCongresso 2011.

8. GODOY CE, et al. Semi-direct technique for partial reconstructions in posterior teeth - case report. Full Dentistry in Science, 2014; 5(17): 124-133.

9. GONÇALVES PE, DEUSDARÁ ST. Lesões cervicais não cariosas na prática odontológica atual: diagnóstico e prevenção. Revista de CiênciasMédicas, 2012; 20(5-6): 145-152.

10. KIM KL, et al. The effect of clinical performance on the survival estimates of direct restorations. Restorative Dentistry \&Endodontics, 2013; 38(1): 11-20.

11. KUMAR S, et al. Prevalence and risk factors for non-carious cervical lesions in children attending special needs schools in India. Journal of Oral Science, 2015; 57(1): 37-43.

12. LUQUE-MARTINEZ I, et al. Effect of EDTA conditioning on cervical restorations bonded with a self-etch adhesive: $A$ randomized double-blind clinical trial. JournalofDentistry, 2015; 43(9): 1175-1183.

13. MARSON FC, et al. Avaliação clínica das lesões cervicais não cariosas. Revista Uningá, 2010; 24(1).

14. MODENA RA, et al. Conhecimento de cirurgiões-dentistas sobre o diagnóstico e o tratamento de lesões cervicais não cariosas: um estudo piloto em rede colaborativa. Revista da Faculdade de Odontologia-UPF, 2016; 21(2): 178-186.

15. MONTEIRO RV, et al. Técnica semidireta: abordagem prática e eficaz para restauração em dentes posteriores. RevistaCiência Plural, 2017; 3(1): 12-21.

16. NASCIMENTO GG, et al. Do clinical experience time and postgraduate training influence the choice of materials for posterior restorations? Results of a survey with Brazilian general dentists. Brazilian Dental Journal, 2013; 24(6): 642646.

17. OLIVEIRA LKM. Ocorrência, severidade e fatores associados às lesões cervicais não cariosas em pacientes sob privação social. Trabalho de Conclusão de Curso (Graduação em Odontologia). Universidade Federal do Rio Grande do Norte, Natal, 2016.

18. PECIE R, et al. Noncarious cervical lesions (NCCL)-A clinical concept based on the literature review. Part 2: Restoration. American Journal of Dentistry, 2011; 24(3): 184-192.

19. PECIE R, et al. Noncarious cervical lesions- A clinical concept based on the literature review. Part 1: Prevention. American Journal of Dentistry, 2011; 24(1): 49.

20. PEREZ CR, et al. Restoration of Noncarious cervical lesions: when, why and how. InternationalJournalofDentistry, 2012; 2012.

21. RODRIGUES PSH, et al. Avaliação clínica de restaurações classe $v$ restauradas com a técnica direta e semidireta.Tese (Doutorado) - Pontifícia Universidade Católica do Rio Grande do Sul, Porto Alegre, 2013.

22. SOUSA LX, et al. Abfração dentária: um enfoque sobre a etiologia e o tratamento restaurador.Archivesof Health Investigation, 2018; 7(2): 51-53.

23. SOUZA FHCD. Efeito da técnica restauradora, do tipo de preparo e do envelhecimento de restaurações de resina composta sobre a resistência à fratura dental, resistência adesiva e vedamento marginal. Tese (Doutorado) Faculdade de Odontologia da Universidade Federal de Pelotas, Pelotas, 2006.

24. SPLIETH CH, TACHOU A. Epidemiology of dentin hypersensitivity. Clinical Oral Investigation, 2013; 17(1): 3-8.

25. SPREAFICO RC, et al. Clinical performance and marginal adaptation of class II direct and semidirect composite restorations over 3.5 years in vivo. JournalofDentistry, 2005; 33(6): 499-507.

26. TONOLLI G, HIRATA R. Técnica de restauração semidireta em dentes posteriores - uma opção de tratamento. Revista da Associacão Paulista de Cirurgiões Dentistas, 2010; 64(1): 90-96.

27. TUNCER D, et al. Avaliação Clínica de 1 Ano de Compósitos Microhíbridos utilizados no Restauração de lesões cervicais não-cariosas. OHDM Ancara, 2014; 13(2): 366-371.

28. TURBINO ML, et al. Restaurações de resina composta semidireta em dentes posteriores.. Pedrosa SF. Pró-Odonto estética: programa de atualização em odontologia estética. Porto Alegre: Artmed/Panamericana Editora, 2011; 99135.

29. VAN DIJKEN JWV. Direct resin composite inlays/onlays: an 11 year follow-up. Journal of Dentistry, 2000; 28(5): 299306

30. VIEIRA RM, et al. Avaliação clínica retrospectiva de restaurações cervicais de resina composta. Revista da Faculdade de Odontologia-UPF, 2013; 18(3): 335-344.

31. ZORBA YO, et al. Comparing the shear bond strength of direct and indirect in relation to different surface conditioning and curing techniques. European Journal of Dentistry, 2013; 7(4): 436-441. 